\title{
The Advances of Eating Disorders in Adolescent
}

\author{
Boyan Meng ${ }^{1, *}$ \\ ${ }^{1}$ University of Minnesota, Twin Cities, Minneapolis, Minnesota, United States \\ "Corresponding author. Email: meng0101@umn.edu
}

\begin{abstract}
This study is a review of a selection of the literature on eating disorders. This study, focusing on anorexia nervosa, includes its prevalence, etiology, impacts, treatments, and recommendations for future development. This study also explores the performance of family therapy for anorexia nervosa in adolescents. The results and discussion section presents the possible causes of eating disorders, such as anorexia nervosa, from biological and social perspectives. The current study also articulates the impact of anorexia nervosas on individuals, social relationships, and social, emotional functioning. Recommendations in the conclusion section provide directions for future research. Overall, the audience will be able to acquire some general knowledge about eating disorders and learn about the efficacy of family therapy with eating disorders.
\end{abstract}

Keywords: eating disorders, anorexia nervosa, family therapy, adolescent.

\section{INTRODUCTION}

According to the Diagnostic and Statistical Manual of Mental Disorders [1], eating disorders are defined as changed consumption of food that significantly damages "physical health or psychosocial functioning" that results from constantly disturbed eating behavior [1]. Anorexia nervosa, as a major component of eating disorders, will be the focus of the present study. This study will focus on the effectiveness of family therapy in treating adolescents with anorexia nervosa. The features of anorexia nervosa include excessive food restriction and weight loss because people with anorexia nervosa have a distorted view of their body image [2]. Many feel out of control with their life and feel comfortable with the control they can exercise over their own body [2].

According to the literature, there is an ever-increasing amount of research on the treatment of adolescent eating disorders [3]. In adolescents, family therapy is a wellestablished and effective treatment for anorexia nervosa [4-8].

According to ANAD [9] and SingleCare [10], there are 70 million people internationally who live with eating disorders, and it affects at least $9 \%$ of the population worldwide. Eating disorders have emerged as having the highest mortality rate of any mental illness [10]. In the United States, approximately 30 million Americans live with an eating disorder [10]. For adolescent females in the United States, eating disorders are the third most common chronic illness [10]. For anorexia nervosa specifically, the prevalence rate is eight per 100,000 per year [11]. The average prevalence rate in young females with anorexia nervous is $1 \%$ [11]. There is a significantly increased rate of anorexia nervosa in 15-24-year-old females [11].

Given the increasing amount of research on eating disorders and prevalence of eating disorders on adolescents with anorexia nervosa, the intent of this study is to 1) to provide an overview of anorexia nervosa with an eating disorder; 2) to understand the effects of anorexia nervosa on individuals and families; 3 ) to provide a comparison between a select number of treatment groups and a control group that received no treatment, and finally, 4) to extend the current literature review to focus on adolescent.

\section{LITERATURE REVIEW}

\subsection{Etiology}

\subsubsection{Biological}

The etiology of eating disorders is complicated. According to the literature, the main risk factors of eating disorders are biological causes and social influences. Firstly, the biological factor is associated with a genetic predisposition. Family studies have shown that firstdegree relatives of people with eating disorders are more likely than other relatives to develop anorexia nervosa 
[12]. Twin studies have demonstrated that the development of anorexia nervosa could largely be attributed to genetic influences with the heritability range from $28 \%-74 \%$ [12]. However, there is no conclusive evidence that specific genes are responsible for the development of anorexia nervosa [12].

\subsubsection{Environmental}

The environmental factors are related to social difficulties and exposure to social media. For example, unpleasant social network experiences and sudden exposure to death are risk factors for an eating disorder [13]. There is also some evidence that unpleasant perinatal events, attachment problems, such as insecure attachment, and temperamental characteristics, such as loneliness, shyness, and sensitivity to shame, increase the risk of developing eating disorders [14]. The abnormal eating behavior and attitude could result from teasing about weight and physical appearance [13]. Persons with eating disorders are deficient in social skills, such as difficulty in knowing others' emotions in social interactions and difficulty in emotional expression. This increases the risk of developing an eating disorder [13]. According to Cardi [13], people with eating disorders experience a lack of social belonging, safety needs, and negative self-worth, which increases their vulnerability to developing eating disorders. The eating disorder symptoms can be attributed to the "critical comments about eating", bullying, comparisons on the body, eating, and exercise as well [13].

Exposure to social media plays a significant role in the development of an eating disorder in adolescents. On the one hand, exposure to social media has a direct relationship with body dissatisfaction. Morris and Katzman [15] have shown that the media burdens adolescent girls with strong desires to be thin. After viewing bodies deemed to be ideal by the media, female adolescents have significantly more negative self-body images than those viewing average body images [15]. On the other hand, the influence of media exposure influences adolescents' weight management behaviors. In adolescent females, several cross-sectioned studies have demonstrated positive correlations between watching beauty and fashion magazines and augmented weight concerns or eating disorder symptoms [15]. Those who read fashion magazines frequently had two times the possibility to have dieted than those who read fashion magazines infrequently [15]. In adolescent females, photo investment and photo manipulation are associated with eating concerns and symptoms of anorexia nervosa [16].

In the study, both adolescent males and females were determined to look like the images in the media, and it is more likely for them to report weight concerns and become constant dieters [15]. In undergraduate college students, abnormal eating symptoms, motivation for thinness, body dissatisfaction, ineffectiveness in women, and personal thinness and restrictive eating in men can be attributed to media exposure [16]. Media exposure is also associated with the development of unhealthy body images, affecting adolescents' perceptions of body shape and body satisfaction [16]. For photo-based social media specifically, according to Santarossa and Woodruff [17], in adolescent males and females, the exposure to photobased social media behaviors, includes avoidance of posting selfies, photo investment, photo manipulation, investment in other's selfies, is associated with the diagnosis of eating disorder symptoms and maladaptive eating attitudes and behaviors. Choosing selfies to post on social media, editing images, and monitoring comments are related to developing an eating disorder [17].

\subsection{Impacts}

\subsubsection{Individual Impact}

One of the most prominent individual impacts of an eating disorder is suicidal behavior. There is a significant increase in rates of suicide attempts in females with all types of eating disorders [18]. For anorexia nervosa specifically, suicide is the second primary attribution to death., As reflected by the statistics, approximately $25 \%$ to $33 \%$ of persons with anorexia nervosa have suicidal thoughts and attempts at suicide [19]. Consistent with this finding, Pisetsky [18] has shown that $78.3 \%$ of people with anorexia nervosa that attempted suicide have the willingness to die. The risk of suicide is increased in women with anorexia nervosa compared with healthy controls. Suppose the women are comorbid with anorexia nervosa and bulimia nervosa, a type of eating disorder that characterized by "recurrent binge eating followed by compensatory behaviors", and anorexia nervosa and purging disorder, a type of eating disorder that characterized by the use of purging to "induce weight loss or manipulate body shape" the risk of suicide increased $[2,18,20]$. Females with anorexia nervosa and bulimia nervosa have the highest rate of suicide attempts and "the highest mean number of lifetime suicide attempts" [18].

Besides the risk of suicide, persons with anorexia nervosa also suffer from personal distress. According to Corstorphine [21], distress tolerance is defined as "the ability to endure and accept negative effects", making problem-solving effective [21]. Poor distress tolerance is associated with high emotional vulnerability and poor emotional regulation. An eating disorder is characterized by emotional regulation difficulties and poor distress tolerance [21]. For example, women with eating disorders are significantly more apt to use emotions as an avoidance strategy than healthy people as controls [9]. In comparison, healthy controls are more possible to utilize problem-solving strategies [21]. Consistent with this result, people with anorexia nervosa showed significantly 
lower emotional awareness and utilized inadequate emotional regulation strategies than normal controls [22]. However, the results for emotional regulation are mixed. According to Gilboa-Schechtman [22], the results are insignificant even though females with eating disorders reported less emotional awareness or poor emotional regulation. When compared people with bulimia nervosa, people with anorexia nervosa have more alexithymia. This term describes "difficulty in experiencing, identifying, and expressing emotions", and less emotional clarity, attention to emotion, significantly more difficulties in emotional awareness, higher levels of emotional dysregulation, a term describes poor emotional regulation. The persons are unable to respond others in a variety of emotional reactions that are traditionally accepted and lower levels of emotional self-efficacy [22, $23,24]$. People with an eating disorder may have more risks to comorbidity with depression and anxiety disorders. For instance, as shown by Gilboa-Schechtman [22], when compared with normal controls, people with eating disorders experienced "significantly higher levels of depression state and trait anxiety and social anxiety" [22].

\subsubsection{Social Impact}

The influences of eating disorders can be extended to social relationships. From the perspective of relationships in schools, a significant number of young women mentioned how their eating disorders, especially weight loss, made them become the central point of conversations [25]. Medical professions, teachers, the family cannot comprehend the symptoms of eating disorders, and adolescent females choose not to express their mental discomforts [25]. Thus, these girls typically do not receive help from others. A substantial number of girls reported being bullied in schools, experience depression, and engage in self-harm to cope with the burden of an eating disorder [25]. Their schools educate them about health issues, such as eating the "right foods", "exercising", and achieving the "right size", and what is "good" and what is "bad" [25]. These experiences in schools create environments that are harmful to the wellbeing of young women with anorexia [25].

Eating disorders also threaten friendships. A majority of the influences of anorexia on making and preserving friendships are negative, such as alienating from their friends, losing friends, and feeling their friends are unable to comprehend eating disorders [26]. One of the most prevalent ways that friendships are destroyed is that people with anorexia simply stop "making time for friends", prioritizing other activities like exercise or food-control activities, rather than making or maintaining friends [26]. People with eating disorders become alienated from their friends and thus results in peer isolation [27]. They cannot get along with their friends and sometimes felt "inferior", "outcast", and "lonely"
[27]. To conform to their friends, people with eating disorders engage in food restrictions [27]. Persons with an eating disorder feel burdened by the eating disorder and feel tired by trying to "keep up their friendships" [27]. Additionally, when persons with eating disorders get admitted to the hospital, they lose connections with their friends [27].

The effects of eating disorders on familial relationships cannot be ignored. If the child is diagnosed with anorexia nervosa, she will significantly change familial relationships [28]. For instance, siblings become acrimonious, and parents become introspective concerning their own contributions to their children's mental illness [29]. An 18-20-year-old daughter is more likely to show greater family relationship changes than those of 14-17-year-olds because parents cannot participate in their recovery due to feelings of frustration and helplessness [28]. For the 23-old-group, the familial relationship does not change much because daughters with eating disorders begin to control their eating disorders themselves [28]. Children with eating disorders could raise quarrels at mealtimes that impact all aspects of the family. Parents find it difficult to comprehend eating disorders and have no idea how to assist their children. They find it difficult to accommodate their child's eating disorder [28, 30]. These negative experiences in families result from various emotions, such as "guilt, anger, anxiety, fear, and denial" and challenges [29].

As well as the collapse of interpersonal relationships, people with eating disorders are also disadvantaged in social, emotional functioning. Persons with anorexia nervosa demonstrate problematic emotional recognition and regulation. For instance, they have difficulties setting personal resolutions, figuring out emotional and mental states, and imitating individuals' emotional expressions; People with anorexia nervosa also have dysfunctional facial expressions, augmented sensitivity to danger, and discriminative reactions towards others [13, 14]. In comparison with normal controls, individuals with eating disorders present more unfavorable social comparisons, more submissive behaviors, and have higher levels of proneness to shame compared to healthy controls [14]. These social difficulties and lack of interpersonal skills affect family and peer relationships, contributing to social isolation and symptom deterioration [13]. Since individuals engage in a vicious cycle, the maintenance of an eating disorder can result from abnormal social and emotional functioning. Individuals with eating disorders have poor emotional regulation, resulting in bullying, verbal and physical abuse [14]. Family members may feel anxiety and avoid these aggressive behaviors, exacerbating the symptoms, diminishing effective caregiving, and mirroring and increasing emotional arousal [14]. This cycle intensifies the social, emotional functioning and eventually leads to disruptive relationships [14]. 


\subsection{Family Therapy}

Given the prevalence of eating disorders, their negative personal and social influences on people, and their paramount impacts on interpersonal relationships, it is imperative to examine the effectiveness of therapies with persons who have an eating disorder. Although there are lots of types of therapies for eating disorders, such as cognitive-behavioral therapy and adolescent-focused therapy, in the last ten years, eating disorder-focused therapy has produced impressive results. The most effective results come from early intervention in the form of family therapy, according to Marzola [31]. Over half to 70 percent of adolescence restore their weight after 12 months of treatment. Throughout the treatment, people with anorexia nervosa show "favorable cognitive remission" [31]. The adolescents with anorexia nervosa who received family therapy had a reduction in hospital admission duration, therefore diminishing relapse and readmission rates [31].

\subsubsection{Definition of family therapy}

Before summarizing the research that has been conducted on eating disorders, it is necessary to first clarify the definition of family therapy in eating disorders. According to Robinson [4], the purposes of family-based therapy (FBT) are to encourage parents to facilitate weight gain in their children, to discontinue the symptoms and problematic eating habits, and to support normal eating patterns and food choices. After children have gained weight, the therapist supports the family to retain control over the eating patterns and food choices [4]. Eventually, the adolescents are offered chances to develop their own identities [4]. It is not only the eating that has to be addressed, as eating is one of the symptoms of anorexia nervosa, so they need therapy as well.

In Jewell [8], researchers provide the readers with another type of family therapy. The first step is "Engagement and development of therapeutic alliance", which is defined as providing general knowledge of anorexia nervosa, including the physiological and psychological effects of starvation, medical examination, and helping the families to communicate with each other without blame and guilt [8]. Secondly, the therapists help families to manage the disorder [8]. For example, the families are encouraged to share their stories at mealtimes, giving parents a sense of autonomy in controlling their children's eating [8]. After children's weight restoration in phase three, "Exploring issues of individual and family development", begins [8]. The goal of therapy is to concentrate on age-appropriate responsibilities, individual needs, and other family members and helping the families to reduce their dependence on therapists [8]. The purposes of the final step, "discussion of future plans and discharge", are to provide support to the family for relapse prevention and tolerance of recovery, discuss the recovery process, and assist children in addressing future difficulties [8].

\subsubsection{Effectiveness of family therapy}

What's the effectiveness of family therapy on anorexia nervosa? Researchers have offered answers to this question. This literature review includes examining what studies have shown about the efficacy of family therapy on adolescent females with anorexia nervosa and comparisons with other treatments.

Cognitive behavioral therapy (CBT) assumes that psychological problems originate from faulty ways of thinking and learning patterns of non-helpful behavior. CBT aims to assist people in altering these thinking patterns [32]. Compared to CBT in adolescents with anorexia nervosa, the effectiveness of family therapy is superior to diminishing binge-eating symptoms [6]. Binge-eating disorder is a type of eating disorder. The main symptom is excessive, uncontrollable, impulsive eating behavior without compensatory behavior [2]. Nevertheless, in older adolescence, there is no demonstrated difference between family therapy and CBT [6, 33].

With individual therapy, such as a type of treatment where the therapists assist only a single individual to address his or her problems, compared to family therapy, family therapy is more effective in the alleviation of eating disorder symptoms than treatment as usual [34]. Specifically, in adolescents with anorexia nervosa, the effectiveness of family therapy is better than that of nonspecific individual therapy [6]. Nevertheless, some studies have shown that in adolescence, there is no significant difference between family therapy and individual therapy [34].

In-patient treatment groups included in-patient hospital stays for several months, and patients with anorexia nervosa also received weekly individual therapy, dietary counseling, and occupational therapy [35]. When compared family therapy with in-patient groups, the in-patient groups gained weight more quickly than family therapy groups at one-year follow-up. However, there is no clear difference in weight gain between the in-patient group and the family psychotherapy group. In adolescents with anorexia nervosa, out-patient, individual and dietary counseling participants gained significantly more weight than that of no treatment group at one-year follow-up [35].

When compared with adolescent-focused therapy (AFT), which is a "developmentally tailored individual therapy", those who receive family therapy have lower hospitalization rates [36]. At the end of the treatment, people with eating disorders who received family therapy improve significantly regarding body mass index (BMI) and experienced bigger changes in eating-related psychopathology. In contrast, these results were not 
presented at follow-up [36]. BMI is defined as an individual's weight in kilograms divided by the square of height in meters. This measurement is important because it categorizes a person as normal weight, underweight, or overweight. Remission rates did not differ significantly for those who received family therapy and AFT, whereas family therapy showed higher remission rates than those who received AFT at 6-12-month follow-up [36].

In adolescents with anorexia nervosa, when compared to short-term family therapy (10 sessions over 6 months) with long-term family therapy (20 sessions over 12 months), short-term family therapy is an effective treatment [37]. However, long-term family therapy produced significantly more desirable results for adolescents with severe and persistent eating-related obsessive-compulsive behavior. This disorder includes repetitive behavior to diminish the anxiety that resulted from intrusive thoughts or from non-intact families. Long-term family therapy produced significantly more desirable results than those of short-term family therapy [37]. Adolescents with anorexia nervosa from intact families take advantage of their parents' investments to help them recover from eating disorders [37].

Family therapy has long-term effectiveness. Nonetheless, its long-term effectiveness differs for the ages of onset of an eating disorder. In a one-year followup, family therapy produced better consequences for patients who begin the eating disorder before 18 years old than individual therapy [5]. Persons accepting family therapy demonstrated significantly more improvement from the perspectives of nutrient status, menstrual function, psychosexual adjustment, and socioeconomic status [5]. More patients receiving family therapy keep their weight at discharge than an individual therapy group, and people receiving family therapy gained more weight than those receiving it [5]. There is no significant difference in weight gain for patients who demonstrate mental illness when older than 18 [5].

At 6-12-month follow-up, family therapy is superior to individual therapy [34]. One explanation is that individuals lose the support of their therapists at the end of treatments. In comparison, family therapy therapists provide the techniques with parents to assist the adolescents in the long term [34]. The other explanation is that with the assistance of families, adolescents still retain weight, whereas those adolescents who receive individual therapy discontinue the weight gains [34].

\section{CONCLUSION}

Even though researchers have researched family therapy within eating disorders, some methodological issues could be improved. One of the problems is the relatively small and biased sample sizes. For instance, many of the studies have a sample size of less than one hundred, include only females, and only include in- patient people with an eating disorder. As a result, the research conclusions may not be generalizable to the male population or out-patient people with eating disorders. Thus, future research should include larger sample sizes and recruit both male and female participants. Future studies should also be conducted in out-patient settings to increase its ecological validity.

Secondly, some of the studies only use objective measures to evaluate the effectiveness of family therapy, such as BMI, a measurement to categorize a person as normal weight, underweight, or overweight and weight. Although this data collection method helps researchers increase internal validity, researchers miss some pertinent qualitative data, such as feelings of people with an eating disorder about family therapy. Some of the studies use self-reporting interviews and questionnaires as their data collection method, limiting its internal validity because participants' recall of events may be inaccurate and subjective, and participants may provide dishonest answers. Therefore, future research on family therapy within eating disorders should use both objective measures and collect self-reporting data from both parents and adolescents, since the combination of these two types of collection methods not only helps the researchers to retain the internal validity but also assists researchers in utilizing multiple sources to confirm the accuracy of the data.

In conclusion, eating disorders are among the most dangerous and prevalent mental disorders worldwide because of the significantly affected populations and high mortality rates. Biological and social factors contribute to the development of eating disorders, whereas more research is needed to discover the specific genes responsible for eating disorders. Eating disorders cause unintended outcomes for individuals who experience eating disorders, such as suicide attempts, low distress tolerance, difficulty maintaining interpersonal relationships, and difficulty in emotional and social functioning. Eating disorders threaten social functioning, as individuals with eating disorders have dysfunctional emotional regulation and poor stress tolerance. Eating disorders destroy relationships within schools, friendships, and familial relationships. Although family therapy is not superior to CBT and in-patient groups, it is more effective than individual therapy. In summary, to avoid negative outcomes, family therapy is a good choice for eating disorder treatment because of its high remission rates, efficacy in helping the persons with anorexia nervosa to increase BMI, and long termeffectiveness, depending on the specific circumstances of the individuals.

\section{REFERENCES}

[1] Feeding and eating disorders (n.d.), DSM Library, https://dsm.psychiatryonline.org/doi/full/10.1176/a ppi.books.9780890425596.dsm10 
[2] Feeding and eating disorders (n.d.), Lumen,https://courses.lumenlearning.com/boundle ss-psychology/chapter/feeding-and-eatingdisorders/

[3] S.B. Murray \& D. Le Grange, D, Family therapy for adolescent eating disorder: An update, in: Current Psychiatry Reports, vol. 16, 2014, pp. 1-7. DOI: 10.1007/s11920-014-0447-y.

[4] A.L. Robinson, J. Dolhanty, J. \& Greenberg, L.,Emotion-focused family therapy for eating disorders in children and adolescents, in: Clinical Psychology and Psychotherapy, vol. 22, 2015, pp. 75-82. DOI: $10.1002 / \mathrm{cpp} .1861$.

[5] G.F.M. Russell, G.I. Szmukler, C. Dare \& I. Eisler, I., An evaluation of family therapy in anorexia nervosa and bulimia nervosa, in: Archives of General Psychiatry, vol. 44, 1987, pp. 1047-1056.

[6] P.K. Keel \& A. Haedt, Evidence-based psychosocial treatments for eating problems and eating disorders, in: Journal of Clinical Child \& Adolescent Psychology, vol. 37, 2008, pp. 39-61. DOI: $10.1080 / 15374410701817832$

[7] S.B. Murray \& D. Le Grange, D, Family therapy for adolescent eating disorder: An update, in: Current Psychiatry Reports, vol. 16, 2014, pp. 1-7. DOI: 10.1007/s11920-014-0447-y.

[8] T. Jewell, E. Blessitt, C. Stewart, M. Simic \& I. Eisler, Family therapy for child andadolescent eating disorders: A critical review, in: Fam Proc, vol. 55, 2016, pp. 577-595. DOI: 10.1111/famp.12242/

[9] Eating disorder statistics (n.d.), ANAD, https://anad.org/get-informed/about-eatingdisorders/eating-disorders-statistics/

[10] Eating disorder statistics 2021 (2021), SingleCare. https://www.singlecare.com/blog/news/eatingdisorder-statistics/

[11] H.W. Hoek, Incidence, prevalence, and mortality of anorexia nervosa and other eating disorders, in: Current Opinion Psychiatry, vol. 19, 2006, pp. 389394. DOI: 10.1097/01.yco.0000228759.95237.78

[12] S.E. Trace, J.H. Baker, E. Penas-Lledo \& C.M. Bulik, C.M, The genetic of eating disorders, in: Annual Review of Clinical Psychology, vol. 9, 2013, pp. 589-620. DOI: 10.1146/annurev-clinpsy050212-185546

[13] V. Cardi, K. Tchanturia, \& J. Treasure, Premorbid and illness-related social difficulties in eating disorders: An Overview of the Literature and Treatment Developments, in: Current
Neuropharmacology, 16, 2018, pp. 1122-1130. DOI: $10.2174 / 1570159 X 16666180118100028$

[14] J. Treasure, F. Cordield \& V. Cardi, A three-phase model of the social emotional functioning in eating disorders, in: European Eating Disorders Review, vol. 20, 2012, pp. 431-438. DOI: 10.1002/erv.2181

[15] A.M. Morris \& D.K. Katzman, D.K, The impact of the media on eating disorders in children and adolescents, in: Paediatr Child Health, vol. 8, 2003, pp. 287-289.

[16] A.R. Lonergan, K. Bussey, J. Fardouly, S. Griffiths, S.B. Murray, P. Hay, J. Mond, N. Trompeter \& D. Mitchison, D, in: International Journal of Eating Disorders, vol. 53, 2020, pp. 755-766. DOI: 10.1002/eat.23256

[17] S. Santarossa \& S.J. Woodruff., \#Socialmedia: Exploring the relationship of social networking sites on body image, self-esteem, and eating disorders. Social Media + Society, 2017, pp. 1-10. DOI: 1: $0.1177 / 2056305117704407$

[18] E. M. Pisetsky, L. M. Thornton, P. Lichtenstein, N.L. Pedersen \& C.M. Bulik. Suicide attempts in women with eating disorders. Journal of Abnormal Psychology, vol. 4, 2013, pp. 1042-1056. DOI: $10.1037 / \mathrm{a} 0034902$

[19] R.A. Smith, K. Zuromski, R.D. \& Dodd, Eating disorders and suicidality: what we know, what we don't know, and suggestions for future research, in: Current Opinion in Psychology, vol. 22, 2018, pp. 63-67. DOI: 10.1016/j.copsyc.2017.08.023

[20] Purging disorder: What is it? (n.d.), healthline, https://www.healthline.com/health/eatingdisorders/purging-disorder

[21] E. Corstorphine, V. Mountford, S. Tomlinson, G. Waller \& M. Caroline, Distress tolerance in eating disorders, in: Eating Behaviors, vol. 8, 2007, pp. 91 97. DOI: $10.1016 /$ j.eatbeh/2006.02.003

[22] E. Gilboa-Schechtman, L. Avnon, E. Zubery, \& P. Jeczmien, Emotional processing in eating disorders: Specific impairment or general distress related deficiency, in: Depression and Anxiety, vol. 23, 2006 pp. 331-339. DOI: 10.1002/da

[23] What is emotional dysregulation (n.d.). WebMD, https://www.webmd.com/mental-health/what-isemotionaldysregulation\#: :text=Emotional\%20dys regulation $\% 20$ is $\% 20 \mathrm{a} \% 20$ term, $\operatorname{mood} \% 20$ swings $\%$ 2C\%20or\%20labile\%20mood.

[24] What to know about alexithymia? (n.d.). MedicalNewsToday,https://www.medicalnewstoda y.com/articles/326451 
[25] E. Rich \& J. Evans, Making sense of eating disorders in schools, in: Disclosure: studies in the cultural politics of education, vo. 26, 2005, pp. 247262. DOI: $10.1080 / 01596300500143211$

[26] E.T. Troscianko, 2019, Anorexia and friendship: What eating disorders do to friends.Psychology Today, https://www.psychologytoday.com/us/blog/hungerartist/201910/anorexia-and-friendship-what-eatingdisorders-do-friends

[27] Eating disorders (young people) (n.d.). healthtalk.org, https://healthtalk.org/eatingdisorders/friends-and-relationships

[28] A.A. Gilbert, S.M. Shaw \& M.K. Notar, The impact of eating disorders on family relationships, in: Eating Disorders, vol. 8, 2006, pp. 331-345. DOI: $10.1080 / 10640260008251240$

[29] A. Schwartz (n.d.). Anorexia impacts the whole family, MentalHelp.net., https://www.mentalhelp.net/blogs/anorexiaimpacts-the-whole-

family/?_cf_chl_jschl_tk_=1bc8349f21cf8c776b 3b5fc743432adb095a4859-1623589389-0-

AbOAMmW7OEOGIuSCnmykZXpRM1Yh9eH8 ZvjNW_nhY02g_PyIZQfwFzhUqDQzkLd2Gndgo MVxKyRGGyK3DemViCUA245gTkIuZPD60p61 v86LKtPp9QBZNIZIgenuo6FQNP5nN_12sfxe38y dnb5Xkp_i6Tk9ctQTyEWrKsmGj7oKXpFZ0sgP QI481J0VtHCpyQxyvJ7szL3ekqHJT9w2dHdKJ51 UZjW6NFJAKL06h5-

S9DLmDZn4rNipForZtXCak60SlvPdVy9CcJe1p6 L_r66X5IGxJ-

z6OZHX6N9MdHcTiqYE6e4iQAxqyyJxOOunO2 Nv02faZkx7INoBuXrN_OM_la0giTLmWMlCgd

MXnm-T4pGLptIuakn1K-

v0ao_TmEj3Yvtub3kMHAgOZ8jsg8zLNfO-

6nKhbvDXkA6O6ASthsEtB_Ff5TMfqhmrNPqkV

xfEFRqbzunBzav41v0uE1njCN5BmMagm-

2c5pm2rWyS1lbCda7Fw0wzZyMvdw8qg

[30] Eating disorders (young people) (n.d.). healthtalk.org, https://healthtalk.org/eatingdisorders/parents-and-family

[31] E. Marzola, S. Knatz, S.B. Murray, R. Rockwell, K. Boutelle, I. Eisler \& W.H. Kaye, Short-term intensive family therapy for adolescent eating disorders: 30-month outcome, in: European Eating Disorders Review, vol. 23, 2015, pp. 210-218. DOI: 10.1002/erv.2353

[32] What is cognitive behavioral therapy? (n.d.). American Psychological Association, https://www.apa.org/ptsd-guideline/patients-andfamilies/cognitive-behavioral
[33] J. Ball \& P. Mitchell, A randomized controlled study of cognitive behavior therapy and behavioral family therapy for anorexia nervosa patients, in: The Journal of Treatment \& Prevention, vol. 12, 2004, pp. 303-314. DOI: 10.1080/10640260490521389

[34] J. Couturier, M. Kimber \& P. Azatmari, Efficacy of family-based treatment for adolescents with eating disorders: A Systematic Review and Meta-Analysis, in: International Journal of Eating Behaviors, vol. 46, 2013, pp. 3-11. DOI: 10/1002/eat/22042

[35] A.H., Crisp, K. Norton, S. Gowers, C. Halek, C. Bowyer, D. Yeldham, G. Levett, \& A. Bhat, A controlled study of effect of therapies aimed at adolescent and family psychopathology in anorexia nervosa, in: The British Journal of Psychiatry, vol. 159, 1991, pp. 325-333. DOI: 10.1192/bhp.159.3.325

[36] J. Lock., Evaluation of family treatment models for eating disorders, in: Current Opinion in Psychiatry, vol. 24, 2011, pp. 274-279. DOI: 10.1097/YCO.0b013e328346f71e

[37] J. Lock, W.S. Agras., S. Bryson \& H.C. Kraemer. A comparison of short- and long- term family therapy for adolescent anorexia nervosa. Journal of American Academy of Child and Adolescent Psychiatry, vol. 44, 2005, pp. 632-639. DOI: 10.1097/01.chi.0000161647.82775.0a 